\title{
ON THE STATIONARY BOUSSINESQ-STEFAN PROBLEM WITH CONSTITUTIVE POWER-LAWS
}

\author{
José Francisco Rodrigues* and José Miguel Urbano† \\ *CMAF/Universidade de Lisboa, Av. Prof. Gama Pinto, 2-1699 Lisboa Codex, Portugal; \\ †CMUC/Universidade de Coimbra, Apartado 3008-3000 Coimbra, Portugal
}

(Received 8 January 1997; in revised form 6 May 1997)

\begin{abstract}
We discuss the existence of weak solutions to a steady-state coupled system between a two-phase Stefan problem, with convection and non-Fourier heat diffusion, and an elliptic variational inequality traducing the non-Newtonian flow only in the liquid phase. In the Stefan problem for the $p$-Laplacian equation the main restriction comes from the requirement that the liquid zone is at least an open subset, a fact that leads us to search for a continuous temperature field. Through the heat convection coupling term, this depends on the $q$-integrability of the velocity gradient and the imbedding theorems of Sobolev. We show that the appropriate condition for the continuity to hold, combining these two powers, is $p q>n$. This remarkably simple condition, together with $q>3 n /(n+2)$, that assures the compactness of the convection term, is sufficient to obtain weak solvability results for the interesting space dimension cases $n=2$ and $n=3$. (C) 1997 Elsevier Science Ltd.
\end{abstract}

Keywords: free boundary problems, Boussinesq-Stefan problem, non-Newtonian flow, thermomechanics of solidification, $p$-Laplacian, variational inequalities

\section{INTRODUCTION}

Several free boundary problems arising from materials processing require appropriate mathematical formulations in order to suggest and to apply adequate numerical methods in concrete engineering problems.

For instance, in modern technology of continuous casting of metals, Czochralski crystal growth systems, laser melting and solidification in welding processes, the mathematical modeling of the corresponding problems of change of phase requires to take into account the convection effects in the liquid phase (cf. for example [1-3]).

In the simplest change of phase problem, where only heat conduction is considered, recent mathematical advances have contributed for a better understanding of the classical Stefan problem (cf. for example [4]). However this model has a limited range of applicability. One of the first extensions of this problem consists in considering only the pure convective mechanism in the liquid zone. Even in the special case of a steady-state situation, which will be the only one considered in this work, the complexity of the problem increases enormously and the complete set of equations leads to very difficult mathematical questions, not only from the computational point of view but also from the mere well-posedness of the problem setting. This is of particular importance when some heuristic approaches are considered from natural simplifications, as it is done very often.

A powerful mathematical tool is the well-known variational approach, which has been successfully applied to Navier-Stokes equations (see [5] for instance), and to other classes of non-linear problems in Mechanics (see [6] or [7]). In particular, for steady-state convection problems with change of phase, where the unknowns are the temperature and the velocity fields, a notion of weak solution, for which an existence result can be obtained, has been introduced in [8] for Newtonian fluids. This approach, that requires the continuity of the temperature field in order to give a precise mathematical sense to the liquid zone, has been extended to the continuous extraction case in [9] and to more general situations, 
allowing non-Newtonian flows, in [10]. Combining this approach with the recent contribution of [11], concerning the solvability of the Stefan problem with prescribed convection and non-linear heat conduction, we are able to extend here the variational method to the case where both the constitutive relations for the heat flux vector and the viscous stress tensor are of the power-law type. The interesting feature is not only the fact that this contains the earlier results of $[10]$ as special cases. It also exhibits a clear relation $(p q>n)$ between the product of the integrability exponents for the gradients of the temperature field $(p>1)$ and of the velocity field $(q>1)$ and the space dimension $n=2$ or 3 , in order to guarantee the continuity of the temperature. In addition, due to technical difficulties related to compactness and the integrability of the inertial term in the variational formulation, we need to assume also $q>3 n /(n+2)$ for the velocity field.

Besides the intrinsic mathematical aspects of these conditions, it remains to discuss the interesting question of their mechanical meaning. Certainly our mathematical restrictions on the exponent $q$, preclude power-laws below or equal to $3 / 2$ and $9 / 5$, for space dimensions two and three, respectively. But, on the other hand, they cover the case of Bingham fluids (see [6]) and several classes of fluids with shear dependent viscosity (cf. [7] and [12], and their references).

The contents of this work are as follows. In Scetion 2, we introduce the constitutive assumptions and the heuristic simplifications in the thermomechanical continuum theory that lead to a free boundary value problem. In Section 3, after introducing the appropriate functional framework for the variational formulation, we state our main result on the existence of at least one weak solution to the Boussinesq-Stefan problem. Sections 4 and 5 explain the mathematical proofs, in particular the method of constructing approximate solutions by regularization and penalization of the solid region; this may suggest an iterative method for numerical computations using, for instance, the finite elements method.

\section{CONSTITUTIVE ASSUMPTIONS}

The general equations governing the flow of an incompressible material with heat transfer in a domain $\Omega \subset \mathbb{R}^{n}$ (in the physical cases $n=2$ or $n=3$ ) are the equations of conservation of mass, momentum and energy. In this work we will be restricted to a steady-state situation and the constant density will be taken $\rho \equiv 1$, through the Oberbeck-Boussinesq hypothesis, so these equations reduce, respectively, to

$$
\begin{aligned}
v_{j, j} & =0 \\
v_{j} v_{i, j} & =\sigma_{i j, j}+f_{i} \\
v_{j} e_{, j} & =r-q_{j, j} .
\end{aligned}
$$

Here, using standard notations, $\mathbf{v}=\left(v_{i}\right)$ is the velocity vector, $\mathbf{x}=\left(x_{i}\right) \in \bar{\Omega}$ denote Eulerian coordinates, $\sigma_{i j}$ are the components of the symmetric stress tensor, $f_{i}$ is a density of forces, including a temperature dependence, $e$ is the specific internal energy of the medium, $\mathbf{q}=\left(q_{i}\right)$ is the heat flux vector and $r$ a density of heat supply, which in general includes also the dissipation effects. We make the convention of summing over repeated indices and, as usual, $v_{i, j}$ means $\partial v_{i} / \partial x_{j}$.

We consider the material in $\Omega$, with two phases, a solid phase corresponding to a region $\Sigma$ and a liquid phase corresponding to a region $\Lambda$. The two phases are separated by a solidification front $\Phi$, which is a priori unknown, thus a free boundary, and bounded by the fixed boundary of the domain $\partial \Omega$. The melting temperature at the interface $\Phi$ is given by a prescribed constant that, after renormalization, we take to be zero.

Introducing the specific constitutive relations of the material, by neglecting the deformation effects, we relate the specific energy $e$ with the temperature $\theta$, by

$$
e(\theta)=b(\theta)+\lambda h(\theta) \text { for } \theta \neq 0,
$$

where $b$ is a given continuous function, $\lambda=[e]_{-}^{+}>0$ is the latent heat of phase transition, with $[\cdot]_{-}^{+}$denoting the jump across the free boundary and $h$ is the Heaviside function. 
The constitutive relation between the heat flux and the temperature is described by the power-law

$$
\mathbf{q}=-k \nabla \theta=-|\nabla \theta|^{p-2} \nabla \theta, \quad 1<p<\infty,
$$

with $k=k(\nabla \theta)$ representing the normalized thermal conductivity. For $p=2,(5)$ reduces to the usual Fourier law.

Then, in the solid region $\Sigma(\theta)=\{\theta<0\}$ and the liquid bulk $\Lambda(\theta)=\{\theta>0\}$, from equation (3) we obtain

$$
\mathbf{v} \cdot \nabla b(\theta)=r(\theta)+\Delta_{p} \theta \quad \text { in } \quad \Sigma(\theta) \cup \Lambda(\theta),
$$

which is the stationary heat equation with convection and where $\Delta_{p} \theta=\nabla \cdot\left(|\nabla \theta|^{p-2} \nabla \theta\right)$ is the $p$-Laplacian operator. In (6) we allow a non-linear heat supply $r=r(\theta)$, which however, due to technical difficulties, does not take into account the term of energy dissipation.

On the free boundary $\Phi$, in addition to the condition $\theta=0$, we obtain the classical Stefan condition (see [4], for example), which represents the balance of heat fluxes

$$
[\mathbf{q}]_{-}^{+} \cdot \mathbf{n}=\left[-|\nabla \theta|^{p-2} \nabla \theta\right]_{-}^{+} \cdot \mathbf{n}=-\lambda \boldsymbol{\alpha} \cdot \mathbf{n} \text { on } \boldsymbol{\Phi}=\{\theta=0\},
$$

where $\mathbf{n}$ is the unit normal to $\Phi$, pointing to the liquid region and $\alpha$ is the given velocity of the free boundary. Clearly, the jump condition (7) only takes into account the convective effect at the interface and neglects the surface stresses.

Finally we must also specify the boundary conditions for the temperature on $\partial \Omega$. We will consider $\partial \Omega$ divided into two components $\Gamma_{\mathrm{D}}$ and $\Gamma_{\mathrm{N}}=\partial \Omega \backslash \Gamma_{\mathrm{D}}$, and take mixed boundary conditions:

$$
\theta=\theta_{\mathbf{D}} \text { on } \Gamma_{\mathbf{D}}
$$

which is a Dirichlet condition, and

$$
-\mathbf{q} \cdot \mathbf{n}=\left(|\nabla \theta|^{p-2} \nabla \theta\right) \cdot \mathbf{n}=\mathrm{g}(x, \theta) \text { on } \Gamma_{\mathrm{N}}
$$

that specifies the value of the conormal derivative, with $\mathrm{g}(x, \theta)$ a given function on $\Gamma_{\mathrm{N}}$ and, here, $\mathbf{n}$ the unit outward normal to $\Gamma_{\mathrm{N}}$.

Since we are considering only a steady-state in equilibrium, the velocity field $\mathbf{v}$ in the solid region $\Sigma(\theta)$ and the fixed boundary $\partial \Omega$ is assumed equal to the velocity of the solid-liquid interface $\Phi$

$$
\mathbf{v}=\boldsymbol{\alpha} \quad \text { on } \quad \Sigma(\theta) \cup \Phi \cup \partial \Omega .
$$

This means, in particular, that the equation of motion (2) is to be considered only inside the liquid bulk and this fact will be of great relevance in the weak formulation, as we will see.

We take $\alpha$ to be a rigid motion and, for technical reasons, we shall assume that it satisfies an additional geometrical restriction:

$$
\mathbf{D}(\boldsymbol{x})=0 \text { and } \boldsymbol{\alpha} \cdot \mathbf{n}=0 \text { on } \Gamma_{\mathrm{N}},
$$

where $\mathbf{D}=\left(D_{i j}\right)$ is the velocity of deformation tensor, with components given by $D_{i j}(\mathbf{v})=\frac{1}{2}\left(v_{i, j}+v_{j, i}\right)$.

We consider an incompressible non-Newtonian fluid, with the constitutive relation

$$
\sigma_{i j}=-p \delta_{i j}+S_{i j}
$$

where $p$ is the pressure and $\mathbf{S}=\left(S_{i j}\right)$ is the viscous stress tensor, so that (2) reads

$$
v_{j} v_{i, j}=-p_{, i}+S_{i j, j}(\theta, \mathbf{v})+f_{i}(\theta) \text { in } \Lambda(\theta) \text {. }
$$

In the Oberbeck-Boussinesq approximation, the changes of temperature within the liquid are taken into account, through the driving forces they produce, in the non-linear term $\mathbf{f}(\theta)$ but are neglected in terms of variation of density. The latter is also relevant to the interface conditions (7) and (10) since they impose the adherence of the fluid to the solid part and the incorporation of the melt into the solid at a rate equal to the growth one. This is the approach we follow here in order to simplify the remarkable mathematical difficulties of a more complete model as, for instance, in [13]. 
In the constitutive relation $\mathbf{S}=\mathbf{S}(\theta, \mathbf{v})$ we shall consider the temperature dependence only through viscosity coefficients $\eta_{k}$, while the dependence on the velocity $\mathrm{v}$ may be of power-law type and given in a quite general form through a potential convex function $\mathscr{F}$ on the second scalar invariant $D_{\mathrm{II}}=\frac{1}{2} \mathbf{D}: \mathbf{D}=\frac{1}{2} D_{i j} D_{i j}$, given by

$$
\mathscr{F}(\theta, \mathbf{v})=\mathscr{F}\left(\theta, D_{\mathrm{II}}(\mathbf{v})\right)=\sum_{k=1}^{n} \eta_{k}(\theta) F_{k}\left(D_{\mathrm{II}}(\mathbf{v})\right)
$$

In general, we shall assume that, for a fixed temperature $\theta=\bar{\theta}, \mathbf{S}$ is an element of the subdifferential of $\mathscr{F}$ at the point $\mathbf{D}(\mathbf{v})$ :

$$
\mathbf{S} \in \partial_{\mathbf{D}} \mathscr{F}(\bar{\theta}, \mathbf{v}) \quad \text { at fixed } \bar{\theta} .
$$

Since, $\partial D_{\mathrm{II}} / \partial D_{i j}=D_{i j}$, by the definition of subdifferential, (14) is equivalent to the inequality

$$
\mathscr{F}(\bar{\theta}, \mathbf{w})-\mathscr{F}(\bar{\theta}, \mathbf{v}) \geqslant S_{i j}\left(D_{i j}(\mathbf{w})-D_{i j}(\mathbf{v})\right), \forall \mathbf{w} .
$$

When all the functions $F_{k}$ in (13) are differentiable, the constitutive relation (14) reduces to

$$
S_{i j}=\left\{\sum_{k=1}^{n} \eta_{k}(\theta) F_{k}^{\prime}\left(D_{\mathrm{II}}(\mathbf{v})\right)\right\} D_{i j}(\mathbf{v})
$$

and we can easily recognize some classigal examples of fluids of the differential type, when we take only one function $F=F_{1}$, given, for $d=D_{\mathrm{II}}(\mathrm{v})$, by:

$$
\begin{aligned}
& F(d)=2 d \quad \text { Newtonian fluid, } \\
& F(d)=\frac{2}{q} d^{q / 2}, \text { non-Newtonian fluid, }
\end{aligned}
$$

which is called a pseudo-plastic fluid if $1<q<2$ and a dilatant fluid if $q>2$ (see also [12], for other examples of fluids with shear dependent viscosity); an expression for generalized non-Newtonian fluids, like

$$
F(d)=2 \int_{0}^{\sqrt{2 d}} \tau \mu(\tau) \mathrm{d} \tau
$$

includes some important asymptotically Newtonian models like the Prandtl-Eyring, Cross, Williamson and Carreau models (see [7] for example).

Another important example of the general law (16) is the case of a Bingham fluid (see [6] for example)

$$
S_{i j}=2 \mu D_{i j}+\gamma D_{i j} / D_{\mathrm{II}}^{1 / 2}, \text { for } D_{\mathrm{II}}>0,
$$

which corresponds to the combination of the linear law of a Newtonian fluid, with viscosity $\mu=\mu(\theta)>0$, with the plasticity threshold characterized by a parameter $\gamma=\gamma(\theta) \geqslant 0$. These fluids, corresponding to the limit non-differentiable case $p=1$ in (18), behave like a rigid body for stresses below the yield limit $\gamma$ and they move like viscous fluids beyond that limit, in particular, if $S_{\mathrm{II}}=\frac{1}{2} S_{i j} S_{i j}$ then $S_{\mathrm{II}}^{1 / 2} \leqslant \gamma$ is equivalent to $D_{i j}=0$. They arise in many industrial processes, particularly in chemical and material engineering applications, for instance in rolling and extrusion processes, where the flows may present rigid zones.

\section{THE VARIATIONAL FORMULATION AND THE EXISTENCE RESULT}

Since classical smooth solutions are not expected, following [8-10], we introduce a notion of weak solution through the variational formulation of the problem. We start by setting the appropriate functional framework, defining the spaces involved. Let $1<p, q<\infty$ and let $U$ be an arbitrary open and bounded subset of $\mathbb{R}^{n}$. Denote with $C_{0}^{\infty}(U)$ the space of infinite differentiable functions with compact support in $U$ and

$$
\mathscr{V}(U)=\left\{\mathrm{v} \in\left[C_{0}^{\infty}(U)\right]^{n}: \nabla \cdot \mathbf{v}=0 \text { in } U\right\} .
$$


We now introduce the norm

$$
\|\mathbf{v}\|_{\mathbf{v}^{q}(U)}=\left\{\int_{U}\left[D_{\mathrm{II}}(\mathbf{v})\right]^{q / 2}\right\}^{1 / q}=\left\|D_{\mathrm{II}}^{1 / 2}(\mathbf{v})\right\|_{L^{q}(U)}
$$

and define the Banach spaces

$$
\begin{aligned}
& \mathbf{V}^{q}(U):=\text { closure of } \mathscr{V}(U) \text { for }\|\cdot\|_{\mathbf{V}^{q}(U)}, \\
& L_{a}^{s}(U):=\text { closure of } \mathscr{V}(U) \text { in }\left[L^{s}(U)\right]^{n} .
\end{aligned}
$$

We remark that for any element $\mathbf{w} \in L_{\sigma}^{s}(U)$, we have, with $s^{\prime}=s /(s-1)$ denoting the dual exponent of $1<s<\infty$,

$$
\int_{U} \mathbf{w} \cdot \nabla \Phi=0, \quad \forall \Phi: \nabla \Phi \in\left[L^{s^{\prime}}(U)\right]^{n}
$$

The relation between the spaces $L_{\sigma}^{s}(U)$ and $V^{q}(U)$ is obtained using the imbedding theorems of Sobolev, that we briefly recall

$$
\begin{gathered}
W^{1, q}(U) \subset L^{s}(U), \quad s \leqslant q n /(n-q) \quad \text { if } \quad 1 \leqslant q<n \\
W^{1, q}(U) \subset L^{\mathbf{s}}(U), \quad s<\infty \quad \text { if } q=n \\
W^{1, q}(U) \subset C^{0, \lambda}(U) \cap L^{\omega}(U), \quad 0 \leqslant \lambda \leqslant 1-n / q \text { if } q>n .
\end{gathered}
$$

These imbeddings are compact provided $s<q n /(n-q)$ and $0 \leqslant \lambda<1-n / q$.

We will assume $\Omega$ to be a bounded domain in $\mathbb{R}^{n}, n=2$ or 3 , with a Lipschitz boundary, $\partial \Omega \in C^{0,1}$, such that $\partial \Omega=\overline{\Gamma_{\mathbf{N}}} \cup \overline{\Gamma_{\mathrm{D}}}$, with $\Gamma_{\mathbf{N}}$ and $\Gamma_{\mathrm{D}}$ relatively open in $\partial \Omega$ and $\int_{\Gamma_{\mathrm{D}}} \mathrm{d} \sigma>0$, where $\sigma$ represents the surface measure over $\partial \Omega$. In particular, we can characterize $V^{q}(\Omega)$ by

$$
\mathbf{V}^{q}(\Omega)=\left\{\mathbf{v} \in\left[W_{0}^{1, q}(\Omega)\right]^{n}: \nabla \cdot \mathbf{v}=0 \text { in } \Omega\right\} .
$$

In addition, Korn's inequality holds (cf. [6]) and the norm (19) is equivalent in $V^{q}(\Omega)$ to the $W_{0}^{1, q}(\Omega)$-norm, i.e. the $L^{q}$-norm of $|\nabla \mathbf{v}|$. Hence, using the inequality of Poincaré, we have

$$
\|\mathbf{v}\|_{\left[L^{q}(\Omega)\right]^{n}} \leqslant C\|\mathbf{v}\|_{\left[W_{0}^{1 \cdot q}(\Omega)\right]^{n}} \leqslant C_{*}\|\mathbf{v}\|_{\mathbf{v}^{q}(\Omega)}, \forall \mathbf{v} \in \mathbf{V}^{q}(\Omega) .
$$

We considered mixed boundary conditions in the thermal problem, so the appropriate space of test functions is

$$
Z_{p}=\left\{\xi \in W^{1, p}(\Omega): \xi=0 \text { on } \Gamma_{\mathbf{D}}\right\}
$$

with the norm $\|\xi\|_{Z_{p}}=\|\nabla \xi\|_{\left[L^{p}(\Omega)\right]^{\mathrm{N}}}$. As a closed subspace of $W^{1, p}(\Omega), Z_{p}$ is a reflexive Banach space for this norm, which is equivalent to the usual norm of $W^{1, p}(\Omega)$, due to the following extension of Poincare inequality, valid for $1 \leqslant p<\infty$ :

$$
\exists c_{0}>0:\|\xi\|_{L^{p}(\Omega)} \leqslant c_{0}\|\nabla \xi\|_{\left[L^{p}(\Omega)\right]^{\mathrm{N}}}, \forall \xi \in Z_{p}
$$

Integrating formally by parts equation (6), with $\xi \in Z_{p}$, and taking into account (7) and (9), we get

$$
\begin{aligned}
\int_{\Omega}|\nabla \theta|^{p-2} \nabla \theta \cdot \nabla \xi+\int_{\Omega}[\mathbf{v} \cdot \nabla b(\theta)] \xi-\int_{\Omega} r(x, \theta) \xi= & \int_{\Phi}\left(\left[|\nabla \theta|^{p-2} \nabla \theta\right]_{-}^{+} \cdot \mathbf{n}\right) \xi \\
& +\int_{\Gamma_{N}}\left(|\nabla \theta|^{p-2} \nabla \theta \cdot \mathbf{n}\right) \xi \\
& =\int_{\Phi}(\lambda \boldsymbol{x} \cdot \mathbf{n}) \xi+\int_{\Gamma_{N}} \mathrm{~g}(x, \theta) \xi .
\end{aligned}
$$

Recalling that, by assumption (11), $\alpha$ is a rigid motion, which implies $\nabla \cdot \alpha=0$ in $\Omega$, and $\boldsymbol{\alpha} \cdot \mathbf{n}=0$ on $\Gamma_{\mathrm{N}}$, and denoting by $\chi_{\Lambda(\theta)}$ the characteristic function of the liquid zone, i.e. $\chi_{\Lambda(\theta)}=1$ in $\Lambda(\theta)$ and $\chi_{\Lambda(\theta)}=0$ in $\Omega \backslash \Lambda(\theta)$, we can write

$$
\int_{\Omega} \chi_{\Lambda(\theta)}(\alpha \cdot \nabla \xi)=\int_{\Lambda(\theta)} \nabla \cdot(\xi \alpha)=\int_{\partial \Lambda(\theta)}(\xi \alpha) \cdot \mathbf{n}=\int_{\Phi}(\alpha \cdot \mathbf{n}) \xi
$$


and also,

$$
\int_{\Omega}[\mathbf{v} \cdot \nabla b(\theta)] \xi=\int_{\Omega}[\nabla \cdot(b(\theta) \mathbf{v})] \xi=-\int_{\Omega}[b(\theta) \mathbf{v}] \cdot \nabla \xi .
$$

Then (22) becomes

$$
\int_{\Omega}\left\{\mid \nabla \theta^{p-2} \nabla \theta-b(\theta) \mathbf{v}-\lambda \alpha \chi_{\Lambda(\theta)}\right\} \cdot \nabla \xi-\int_{\Omega} r(x, \theta) \xi=\int_{\Gamma_{N}} \mathbf{g}(x, \theta) \xi, \forall \xi \in Z_{p} .
$$

As usual in the weak formulation for the Stefan problem, we replace the characteristic function $\chi_{\mathrm{\Lambda}(\theta)}$, in the above equation, by a function $\chi$ which is in the maximal monotone graph $H(\theta)$, associated with the Heaviside function, i.e. $\chi=1$ in $\Lambda(\theta)=\{\theta>0\}$ and $\chi=0$ in $\Sigma(\theta)=\{\theta<0\}$. We allow, in this way, a possible mushy region at $\{\theta=0\}$, where $\chi$ may take any value between 0 and 1 .

Turning to the equation of motion and assuming again that the liquid zone $\Lambda(\theta)$ is sufficiently smooth, we integrate (12) by parts with $v_{i}-w_{i}$, where $\mathbf{w}=\left(w_{i}\right)$ is a smooth solenoidal vector field, such that $\mathbf{w}=\mathbf{v}=\alpha$ on $\partial \Lambda(\theta)$, and obtain

$$
\int_{\Lambda(\theta)} S_{i j}\left(w_{i, j}-v_{i, j}\right)+\int_{\Lambda(\theta)} v_{j} v_{i, j}\left(w_{i}-v_{i}\right)=\int_{\Lambda(\theta)} f_{i}(\theta)\left(w_{i}-v_{i}\right)
$$

By the symmetry of $S_{i j}$ and the constitutive law (15), we get

$$
\int_{\Lambda(\theta)} \mathscr{F}(\theta, \mathbf{w})-\int_{\Lambda(\theta)} \mathscr{F}(\theta, \mathbf{v})+\int_{\Lambda(\theta)} v_{j} v_{i, j}\left(w_{i}-v_{i}\right) \geqslant \int_{\Lambda(\theta)} f_{i}(\theta)\left(w_{i}-v_{i}\right),
$$

which is a variational inequality for $v=\left(v_{i}\right)$ in $\Lambda(\theta)$. Introducing the translated velocity

$$
\mathbf{u}=\mathbf{v}-\boldsymbol{\alpha},
$$

we rewrite the variational inequality for $\mathbf{u}$ with an arbitrary $\Psi=\mathbf{w}-\alpha \in \mathbf{V}^{q}(\Lambda(\theta))$ :

$$
\int_{\Lambda(\theta)} \mathscr{F}(\theta, \Psi)-\int_{\Lambda(\theta)} \mathscr{F}(\theta, \mathbf{u})+\int_{\Lambda(\theta)}\left(u_{j}+\alpha_{j}\right) u_{i, j}\left(\Psi_{i}-u_{i}\right) \geqslant \int_{\Lambda(\theta)} f_{i}(\theta)\left(\Psi_{i}-u_{i}\right)
$$

Finally, integrating formally by parts the convection term, we have

$$
\int_{\Lambda(\theta)}\left(u_{j}+\alpha_{j}\right) u_{i, j}\left(\Psi_{i}-u_{i}\right)=\int_{\Lambda(\theta)}\left(u_{j}+\alpha_{j}\right) u_{i} \Psi_{i, j}=\int_{\Lambda(\theta)} \beta(\mathbf{u}, \mathbf{u}, \Psi)
$$

where we define

$$
\beta(\mathbf{u}, \mathbf{v}, \mathbf{w})=-\left(u_{j}+\alpha_{j}\right) v_{i} w_{i, j} .
$$

We call attention to the fact that this function is integrable over any open subset $U$, for $\mathbf{w} \in \mathbf{V}^{q}(U)$, provided the product $u_{j} v_{i}$ belongs to $L^{q^{\prime}}(U)$.

Before presenting the definition of weak solution we remark that a proper variational formulation requires that the liquid zone $\Lambda(\theta)=\{\theta>0\}$ is at least an open subset of $\Omega$, even without any smoothness requirements. This fact is implied by the lower semicontinuity of the temperature, but here we shall demand a little more, by requiring a continuous temperature in the definition.

Definition. We say that the triple $(\theta, \chi, \mathbf{u})$ is a weak solution of $(6)-(7)$, with boundary conditions (8)-(9), and (12)-(14) with (10), for $\mathbf{v}$ given by (24) and $\Lambda(\theta)=\{x \in \Omega: \theta(x)>0\}$ and $\Sigma(\theta)=\{x \in \Omega: \theta(x)<0\}$, if

$$
\begin{gathered}
\theta \in W^{1, p}(\Omega) \cap C^{0}(\Omega), \quad \theta=\theta_{\mathrm{D}} \text { on } \Gamma_{\mathrm{D}} \\
\chi \in L^{\infty}(\Omega), \quad 0 \leqslant \chi_{\Lambda(\theta)} \leqslant \chi \leqslant 1-\chi_{\Sigma(\theta)} \leqslant 1 \text { a.e. in } \Omega \\
\mathbf{u} \in \mathbf{V}^{q}(\Omega), \quad \mathbf{u}=0 \text { a.e. in } \Sigma(\theta) ; \\
\int_{\Omega}\left\{\mid \nabla \theta^{p-2} \nabla \theta-b(\theta)[\mathbf{u}+\alpha]-\lambda \chi \alpha\right\} \cdot \nabla \xi-\int_{\Omega} r(\theta) \xi=\int_{\Gamma_{N}} \mathbf{g}(\theta) \xi, \quad \forall \xi \in Z_{p} ; \\
\int_{\Lambda(\theta)} \mathscr{F}(\theta, \Psi)-\int_{\Lambda(\theta)} \mathscr{F}(\theta, \mathbf{u})+\int_{\Lambda(\theta)} \beta(\mathbf{u}, \mathbf{u}, \Psi) \geqslant \int_{\Lambda(\theta)} \mathbf{f}(\theta) \cdot(\Psi-\mathbf{u}), \forall \Psi \in \mathbf{V}^{q}(\Lambda(\theta)) .
\end{gathered}
$$


Remark 1. The integrability of the convective term in the variational inequality requires a restriction on the admissible values of $q$. In fact, using the Sobolev imbedding, $\mathbf{u} \in\left[\mathrm{L}^{q n /(n-q)}(\Omega)\right]^{n}$ and since $\nabla \Psi \in\left[L^{q}(\Omega)\right]^{n \times n}$ we must have $2(n-q) / q n+1 / q \leqslant 1$, i.e. $q \geqslant$ $3 n /(n+2)$.

Remark 2. The free boundary $\Phi$ is absent from this weak formulation but can be recovered a posteriori as the level set

$$
\Phi=\{x \in \Omega: \theta(x)=0\}=\partial \Lambda \cap \partial \Sigma .
$$

If the $n$-dimensional Lebesgue measure of $\Phi$ is zero, from (26) we see that, in fact, $\chi=\chi_{\Lambda(\theta)}=1-\chi_{\Sigma(\theta)}$. If, in addition, $\Phi$ is a Lipschitz surface, from (28) we recover the Stefan condition (7), and, from (27), also the boundary condition $u=0(v=a)$ on $\Phi$. For these facts, we can look at (27) as a weak formulation for the free boundary conditions of the velocity.

In order to obtain existence of solution we use the following set of quite general assumptions on the functions and the data, for given $1<p, q<\infty$, with $p^{\prime}=p /(p-1)$ :

(A1) $\eta_{k} \in C^{0}(\mathbb{R}), F_{k} \in C^{0}\left(\mathbb{R}^{+}\right), \eta_{k}, F_{k} \geqslant 0, F_{k}(0)=0, \quad \forall k=1, \ldots, n$;

(A2) each $F_{k}$ is convex and $F_{k}(d) \leqslant C\left(d^{q / 2}+1\right)$, for some $C>0$;

(A3) $\exists k^{*}, C_{*}>0, n_{*}>0: \begin{cases}F_{k^{*}}(d) \geqslant C_{*} d^{q / 2}, & \forall d>0 ; F_{k^{*}} \text { is strictly convex, } \\ \eta_{k^{*}}(t) \geqslant \eta_{*}, & \forall t \in \mathbb{R} ;\end{cases}$

(A4) $b: \mathbb{R} \rightarrow \mathbb{R}, \mathbf{f}: \mathbb{R}^{+} \rightarrow \mathbb{R}^{n}$ are continuous functions and $\mathbf{f}(0)=0$;

(A5) $r: \Omega \times \mathbb{R} \rightarrow \mathbb{R}$ is such that $r(x, \cdot)$ is continuous and decreasing for each $x \in \Omega$;

(A6) $r(\cdot, t) \in L^{p^{\prime}}(\Omega)$, for each $t \in \mathbb{R}$;

(A7) $\exists M>0: r(x, t) t \leqslant 0$ for $|t| \geqslant M$, a.e. $x \in \Omega$;

(A8) $\mathrm{g}: \Gamma_{\mathrm{N}} \times \mathbb{R} \rightarrow \mathbb{R}$ is such that $\mathrm{g}(x, \cdot)$ is continuous and decreasing for each $x \in \Gamma_{\mathrm{N}}$;

(A9) $\mathrm{g}(\cdot, t) \in L^{p^{\prime}}\left(\Gamma_{\mathrm{N}}\right)$, for each $t \in \mathbb{R}$;

(A10) $\exists M>0: \mathrm{g}(x, t) t \leqslant 0$ for $|t| \geqslant M$, a.e. $x \in \Gamma_{\mathrm{N}}$;

(A11) $\theta_{\mathbf{D}} \in W^{1, p}(\Omega)$ and $\left\|\theta_{\mathrm{D}}\right\|_{L^{x}(\Omega)} \leqslant M$.

Theorem. Under the assumptions (A1)(A11) and (11), and for each $q>\max \left\{\frac{n}{p}, \frac{3 n}{n+2}\right\}$, there exists at least one weak solution for the Boussinesq-Stefan problem, in the sense of the Definition (25)-(29), such that, in addition, the temperature satisfies the estimate

$$
\|\theta\|_{L^{\infty}(\Omega)} \leqslant M .
$$

Remark 3. From now on, we will consider only the cases $n>1$ and $1<p, q \leqslant n$. The first restriction excludes the trivial case $n=1$, for the sake of uniformity of writing. The second restriction focuses the attention on the cases for which the continuity of the temperature is not an immediate consequence of Sobolev imbedding theorem $(p>n)$ or the fact that $v \in L^{\infty}(q>n)$. Observe that the excluded exponents trivially satisfy the condition $p q>n$.

Remark 4. Our result extends for any $1<p<\infty$, the results of [10] for $p=2$. In fact, for $p=2$ we have $\frac{n}{2}<\frac{3 n}{n+2}$ for the physical cases $n=2$ and $n=3$, and, from our condition $q>\max \left\{\frac{n}{n}, \frac{3 n}{n+2}\right\}$, we recover $q>\frac{3}{2}$ for $n=2$ and $q>\frac{9}{5}$ for $n=3$.

The proof, that we postpone to Section 5, consists of passing to the limit in an approximated problem that we study in the next section.

\section{EXISTENCE OF APPROXIMATE SOLUTIONS}

In this section, we consider an approximate problem and study it from the point of view of the existence of solutions. We also obtain some a priori estimates that will allow us to pass to the limit. We follow the approach of $[8,9]$ and $[10]$, where a penalization of the solid zone is combined with the regularization of the Heaviside graph and of some data.

For each $\varepsilon>0$, we define the continuous function $\chi_{\varepsilon}: \mathbb{R} \rightarrow \mathbb{R}$, given by

$$
\chi_{\varepsilon}(\tau)= \begin{cases}1 & \text { if } \tau<-\varepsilon \\ -1-\frac{2 \tau}{\varepsilon} & \text { if }-\varepsilon \leqslant \tau \leqslant-\varepsilon / 2 \\ 0 & \text { if } \tau>-\varepsilon / 2\end{cases}
$$


set $r_{\varepsilon}(\cdot, \mathrm{t})=r(\cdot, \mathrm{t})-\varepsilon t$ and choose a family of functions $\boldsymbol{\theta}_{\varepsilon} \in C^{0,1}(\Omega)$, uniformly bounded in $W^{1, p}(\Omega)$ and whose traces converge to $\theta_{\mathrm{D}}$ in $W^{1-1 / p, p}\left(\Gamma_{\mathrm{D}}\right)$ as $\varepsilon \rightarrow 0$. We take also a family of $b_{\varepsilon} \in C^{0,1}(\mathbb{R})$ such that $b_{\varepsilon} \rightarrow b$ uniformly on compact sets as $\varepsilon \rightarrow 0$.

We now consider the following approximated problem:

Problem $\left(P_{\varepsilon}\right)$ : Find $\theta_{\varepsilon} \in C^{0}(\Omega) \cap W^{1, p}(\Omega),\left.\theta_{\varepsilon}\right|_{\Gamma_{\mathrm{D}}}=\mathscr{\theta}_{\varepsilon}$, and $\mathbf{u}_{\varepsilon} \in \mathbf{V}^{q}(\Omega)$, such that

$$
\begin{gathered}
\int_{\Omega}\left\{\left|\nabla \theta_{\varepsilon}\right|^{p-2} \nabla \theta_{\varepsilon}-b_{\varepsilon}\left(\theta_{\varepsilon}\right)\left(\mathbf{u}_{\varepsilon}+\alpha\right)-\lambda\left[1-\chi_{\varepsilon}\left(\theta_{\varepsilon}\right)\right] \alpha\right\} \cdot \nabla \xi \\
=\int_{\Omega} r_{\varepsilon}\left(\theta_{\varepsilon}\right) \xi+\int_{\Gamma_{N}} \mathbf{g}\left(\theta_{\varepsilon}\right) \xi, \forall \xi \in Z_{\boldsymbol{p}} \\
\int_{\Omega} \mathscr{F}\left(\theta_{\varepsilon}, \Psi\right)-\int_{\Omega} \mathscr{F}\left(\theta_{\varepsilon}, \mathbf{u}_{\varepsilon}\right)+\int_{\Omega} \beta\left(\mathbf{u}_{\varepsilon}, \mathbf{u}_{\varepsilon}, \Psi\right)+\frac{1}{\varepsilon} \int_{\Omega} \chi_{\varepsilon}\left(\theta_{\varepsilon}\right) \mathbf{u}_{\varepsilon} \cdot\left(\Psi-\mathbf{u}_{\varepsilon}\right) \\
\geqslant \int_{\Omega} \mathbf{f}\left(\theta_{\varepsilon}\right) \cdot\left(\Psi-\mathbf{u}_{\varepsilon}\right), \quad \forall \Psi \in \mathbf{V}^{q}(\Omega) .
\end{gathered}
$$

Before showing that the approximated problem is solvable, we need some auxiliary results. We start with a class of elliptic variational inequalities that is studied in [10]. Consider a non-negative continuous function $a \in C^{0}\left(\mathbb{R}^{n}\right)$ and take $\mathscr{F}, \beta$ and $\mathbf{f}$ as before. For $\tau \in L^{\infty}(\Omega)$ and $\mathbf{w} \in L_{\sigma}^{r}(\Omega)$, for some $r \geqslant 2$, find $\mathbf{u} \in \mathrm{V}^{q}(\Omega)$, such that

$$
\int_{\Omega} \mathscr{F}(\tau, \mathbf{v})-\int_{\Omega} \mathscr{F}(\tau, \mathbf{u})+\int_{\Omega} \beta(\mathbf{w}, \mathbf{u}, \mathbf{v})+\int_{\Omega}[a(\tau) \mathbf{u}-\mathbf{f}(\tau)] \cdot(\mathbf{v}-\mathbf{u}) \geqslant 0, \forall \mathbf{v} \in \mathbf{V}^{a}(\Omega) .(33)
$$

Proposition 1. There exists a unique solution $\mathrm{u} \in \mathrm{V}^{q}(\Omega)$ to the variational inequality (33), with $q>\frac{2 n}{n+2}$ and $r \geqslant \max \left(\frac{n q}{n q+q-2 n}, 2\right)$. Moreover, if there exists $M>0$ such that $\left\|\tau_{\delta}\right\|_{L^{\infty}(\Omega)} \leqslant M$ and

$$
\tau_{\delta} \rightarrow \tau_{0} \text { in } L^{1}(\Omega) \text { and } \mathbf{w}_{\delta} \rightarrow \mathbf{w}_{0} \text { in } L_{\sigma}^{r}(\Omega) \text {-weak, }
$$

then the respective solutions $\mathbf{u}_{\delta}=\mathbf{u}\left(\tau_{\delta}, \mathbf{w}_{\delta}\right)$ and $\mathbf{u}_{0}=\mathbf{u}\left(\tau_{0}, \mathbf{w}_{0}\right)$ satisfy

$$
\mathbf{u}_{\delta} \rightarrow \mathbf{u}_{0} \text { in } \mathbf{V}^{q}(\Omega) \text {-weak and in } L_{\sigma}^{s}(\Omega) \text {-strong, }
$$

for any $s<\frac{n q}{n-q}$ if $q<n$ or any $s<\infty$ if $q \geqslant n$.

Remark 5. We observe at this point that, as a consequence of assumptions (A1)-(A2), for each $\tau \in L^{\infty}(U)$, the functional

$$
\mathscr{I}_{U}(\tau, \mathbf{v})=\int_{U} \mathscr{F}(\tau, \mathbf{v})=\sum_{k=1}^{n} \int_{U} \eta_{k}(\tau) F_{k}\left(D_{\mathrm{II}}(\mathbf{v})\right)
$$

is convex, continuous and non-negative over $V^{q}(U)$. Additionally, (A3) implies that $\mathscr{J}_{U}$ is strictly convex and coercive in the sense that:

$$
\mathscr{J}_{U}(\tau, \mathbf{v}) \geqslant C_{*} \eta_{*}\|\mathbf{v}\|_{\mathbf{v}^{q}(U)}^{q}, \quad \forall \mathbf{v} \in \mathbf{V}^{q}(U) .
$$

Remark 6. In [10], a continuous dependence result is obtained assuming $\tau_{\delta} \rightarrow \tau_{0}$ in $L^{\infty}(\Omega)$. However, from the proof of Proposition 6.1 of [10], it is easily seen that, in fact, the $L^{1}(\Omega)$ convergence is sufficient, provided $\tau_{\delta}$ belong to a bounded subset of $L^{\infty}(\Omega)$, as it is assumed in Proposition 1.

Remark 7. It can be shown, putting $\mathbf{v}=0$ in (33) for $\mathbf{u}_{\delta}$ and using the estimate in Remark 5 , that the following $a$ priori (independent of $\delta$ ) estimate holds

$$
\left\|\mathbf{u}_{\delta}\right\|_{\mathbf{v}^{q}(U)} \leqslant C_{*},
$$

as a consequence of

$$
C_{*} \eta_{*}\left\|\mathbf{u}_{\delta}\right\|_{\mathbf{v}^{q}(\mathbf{U})}^{q} \leqslant \int_{\Omega} \mathscr{F}\left(\tau_{\delta}, \mathbf{u}_{\delta}\right) \leqslant \int_{\Omega} \mathbf{f}\left(\tau_{\delta}\right) \cdot \mathbf{u}_{\delta} \leqslant C\left\|\mathbf{u}_{\delta}\right\|_{L^{1}(\Omega)} \leqslant C\left\|\mathbf{u}_{\delta}\right\| \mathbf{v}^{\mathbf{q}^{(}(U)} .
$$


In fact, the estimate shows more: that the solution of (33) is independent of the choice of $\mathbf{w} \in L_{\sigma}^{r}(\Omega)$ and depends only on $\tau$ through $\|\mathbf{f}(\tau)\|_{L^{\infty}(\Omega)}$.

Another useful result concerns a class of convection-diffusion problems originally considered in [10] only in the case $p=2$ and later extended in [11] for any $1<p<\infty$.

With the previous notations, it consists on determining $\theta \in W^{1, p}(\Omega)$, such that $\theta=\theta_{\mathbf{D}}$ on $\Gamma_{\mathrm{D}}$, and

$$
\int_{\Omega}|\nabla \theta|^{p-2} \nabla \theta \cdot \nabla \xi-\int_{\Omega} r(x, \theta) \xi-\int_{\Gamma_{\Gamma}} \mathrm{g}(x, \theta) \xi=\int_{\Omega} W(x, \theta) \cdot \nabla \xi, \quad \forall \xi \in Z_{p} .
$$

The convective term $W$ is supposed to satisfy the following assumptions:

(B1) $W: \Omega \times \mathbb{R} \rightarrow \mathbb{R}^{N}$ is a Carathéodory function;

(B2) $\operatorname{sign}(t) \int_{\Omega} W(y, t) \cdot \nabla \xi \mathrm{d} y \leqslant 0, \quad \forall \xi \in Z_{p}: \xi \geqslant 0$ in $\Omega, \forall t \in \mathbb{R}:|t| \geqslant M$;

(B3) $\exists w_{0} \in L^{s}(\Omega), s>\frac{n}{p-1}$, such that $|W(y, t)| \leqslant w_{0}(y)$, a.e. $y \in \Omega, \forall t \in[-M, M]$;

(B4) $\exists k \in L^{p^{\prime}}(\Omega)$ such that $|W(y, t)-W(y, s)| \leqslant k(y)|t-s|$, a.e. $y \in \Omega, \forall t, s \in[-M, M]$.

Proposition 2. For any $W$ satisfying (B1)-(B4), and $r$, $g$ and $\theta_{\mathrm{D}}$ satisfying (A5)-(A11), there exists a unique solution to the problem (35), which, in addition satisfies

$$
\|\theta\|_{L^{\infty}(\Omega)} \leqslant M .
$$

Moreover, the solution is locally Hölder continuous and satisfies the estimate, for some exponent $0<\gamma<1, \gamma=\gamma(n, p, s)$, and any compact subset $K$ of $\Omega$,

$$
\|\theta\|_{C^{0, y}(K)} \leqslant C,
$$

where the constant $C=C\left(K, M,\|r(\cdot, M)\|_{L^{\prime}(\Omega)},\left\|w_{0}\right\|_{L^{s}(\Omega)}\right)$.

Proof. For the existence, uniqueness and the a priori estimate in $L^{\infty}$, see [11]. Concerning the Hölder character of the solution, we use the results on the regularity of weak solutions of quasi-linear equations. In our case, we may conclude (37) locally from Theorem 2.1 (p. 441) of [14]. We remark that it is precisely the integrability condition (B3), sharper than the one used in [11], where $W(\cdot, t) \in L^{p^{\prime}}$ only, that allows us to apply these results on the continuity of the solution.

Remark 8. The uniqueness in the case $p>2$ requires the additional assumption that $r$ is a strictly decreasing function.

We recall also that $\frac{n}{p-1} \geqslant p^{\prime}$, since we are considering $p \leqslant n$, in this auxiliary problem.

We will also need a continuous dependence result that was not contained in [11] and that we prove here for the sake of the completeness.

Proposition 3. For any $a, b \in C^{0,1}(\mathbb{R})$, a satisfying $(11)$ and $w \in L_{\sigma}^{*}(\Omega)$, with $r>\frac{n}{p-1}$,

$$
W(x, t)=\lambda a(t) \alpha(x)+b(t)[\mathbf{w}(x)+\alpha(x)]
$$

satisfies assumptions (B1)-(B4).

Moreover, if $\mathbf{w}_{\eta} \rightarrow \mathrm{w}_{0}$ in $L_{\sigma}^{r}(\Omega)$-weak, then the corresponding solutions of (35) $\theta_{\eta}=$ $\theta\left(\mathbf{w}_{\eta}\right) \longrightarrow \theta_{0}=\theta\left(\mathbf{w}_{0}\right)$ in $W^{1 . p}(\Omega)$-weak.

Proof. Assumptions (B1)-(B4) are easily checked by recalling (20) and the assumptions concerning $\boldsymbol{\alpha}$.

Concerning the continuous dependence result, let $\mathbf{w}_{\eta} \rightarrow \mathbf{w}_{0}$ in $L_{\sigma}^{r}(\Omega)$-weak. For simplicity, we consider only the case $\theta_{\mathrm{D}}=0$, i.e. $\theta \in Z_{p}$. Putting $\xi=\theta_{\eta}$ in equation (35) corresponding 
to $\theta_{\eta}$, we obtain

$$
\begin{aligned}
\int_{\Omega}\left|\nabla \theta_{\eta}\right|^{p}-\int_{\Omega} r\left(\theta_{\eta}\right) \theta_{\eta}-\int_{\Gamma_{N}} \mathrm{~g}\left(\theta_{\eta}\right) \theta_{\eta} & =\int_{\Omega}\left\{a\left(\theta_{\eta}\right)\left[\mathbf{w}_{\eta}+\alpha\right]-\lambda b\left(\theta_{\eta}\right) \alpha\right\} \cdot \nabla \theta_{\eta} \\
& =\int_{\Omega} \mathbf{w}_{\eta} \cdot \nabla A\left(\theta_{\eta}\right)-\int_{\Omega} \alpha \cdot \nabla B\left(\theta_{\eta}\right)=0,
\end{aligned}
$$

with $A(t)=\int_{0}^{t} a(\tau) \mathrm{d} \tau$ and $B(t)=\int_{0}^{t}[a(\tau)-\lambda b(\tau)] \mathrm{d} \tau$. Then from the assumptions on $r$ and $\mathrm{g}$, we get

$$
\left\|\theta_{\eta}\right\|_{Z_{p}}^{p}=\int_{\Omega} r\left(\theta_{\eta}\right) \theta_{\eta}+\int_{\Gamma_{N}} \mathrm{~g}\left(\theta_{\eta}\right) \theta_{\eta} \leqslant C\left\|\theta_{\eta}\right\|_{Z_{p}}
$$

and consequently the a priori estimate (independent of $\eta$ ),

$$
\left\|\theta_{\eta}\right\|_{Z_{p}} \leqslant C^{\prime}
$$

Then for an appropriate subsequence $\theta_{\eta} \rightarrow \theta$ in $Z_{p}$-weak, with $\theta \in Z_{p}$. We can pass to the limit in equation (35) corresponding to $\theta_{\eta}$, obtaining that $\theta$ is a solution of (35) corresponding to $w_{0}$ and the uniqueness gives us $\theta=\theta_{0}$ and the whole sequence converges to $\theta_{0}$. This passage to the limit in the equation requires some justification. For each $\xi \in Z_{p}$, put

$$
\left\langle f_{\eta}, \xi\right\rangle=\int_{\Omega} W\left(x, \theta_{\eta}\right) \cdot \nabla \xi \quad \text { and }\langle f, \xi\rangle=\int_{\Omega} W(x, \theta) \cdot \nabla \xi .
$$

Using (B3) and the properties of the Nemytskii operator

$$
\begin{aligned}
G: L^{1}(\Omega) & \rightarrow\left[L^{p^{\prime}}(\Omega)\right]^{\mathrm{N}} \\
\tau & \mapsto G \tau \text { with } \quad G \tau(x)=W^{M}(x, \tau(x)),
\end{aligned}
$$

we obtain $f_{\eta} \rightarrow f$ in $Z_{p}^{\prime}$-strong since $\theta_{\eta} \rightarrow \theta$ in $Z_{p}$-weak hence in $L^{1}(\Omega)$-strong. Since equation (35) corresponding to $\theta_{\eta}$ can be written in the form

$$
A \theta_{\eta}=f_{\eta} \text { in } Z_{p}^{\prime} \text {, }
$$

where $A: Z_{p} \rightarrow Z_{p}^{\prime}$, is defined for $\sigma, \xi \in Z_{p}$, by

$$
\langle A \sigma, \xi\rangle=\int_{\Omega}|\nabla \sigma|^{p-2} \nabla \sigma \cdot \nabla \xi-\int_{\Omega} r(x, \sigma) \xi-\int_{\Gamma_{\mathrm{N}}} \mathrm{g}(x, \sigma) \xi,
$$

we need only show that $A \theta=f$. Since $A$ is a strictly monotone operator we do it using the usual technique (see [15]). The general case is proved in a similar way by considering the translation $\theta_{\eta}-\theta_{\mathrm{D}} \in Z_{p}$.

We can now prove that the approximated problem has at least one solution.

Proposition 4. If $q>\max \left(\frac{n}{p}, \frac{3 n}{n+2}\right)$ then, for each $\varepsilon>0$, there exists at least a solution $\left(\theta_{\varepsilon}, \mathbf{u}_{\varepsilon}\right) \in\left[C^{0, \gamma}(\Omega) \cap W^{1, p}(\Omega)\right] \times \mathbf{V}^{q}(\Omega)$ to the problem $\left(P_{\varepsilon}\right)$, for some $0<\gamma<1$. Moreover the following (independent of $\varepsilon$ ) estimates hold

$$
\begin{gathered}
\left\|\theta_{\varepsilon}\right\|_{W^{1, p}(\Omega)} \leqslant C ; \quad\left\|\theta_{\varepsilon}\right\|_{L^{\infty}(\Omega)} \leqslant M ; \\
\left\|\theta_{\varepsilon}\right\|_{C^{0, \gamma(K)}} \leqslant C(K), \quad \text { for any compact subset } K \subset \Omega ; \\
\left\|\mathbf{u}_{\varepsilon}\right\|_{\mathbf{v}(\Omega)} \leqslant C .
\end{gathered}
$$

Proof. We apply Schauder's fixed point theorem to a non-linear operator $\mathscr{T}$, defined on a ball of $L_{\sigma}^{r}(\Omega)$, where $r>\max \left(\frac{n}{p-1}, \frac{3 n}{n-1}\right)$.

Given $\mathbf{w} \in L_{\sigma}^{r}(\Omega)$, we put

$$
W(x, t)=\lambda\left[1-\chi_{\varepsilon}(t)\right] \alpha(x)+b_{\varepsilon}(t)[w(x)+\alpha(x)],
$$

and define $\tau_{\varepsilon}=\tau_{\varepsilon}(w)$ as the unique solution of (35), corresponding to this choice of $W$ and with $r \equiv r_{\varepsilon}$ and $\theta_{D} \equiv \partial_{\varepsilon}$, given by Proposition 2 . It is easy to see that $W$ is as in 
Proposition 3. We know that $\tau_{\varepsilon} \in W^{1, p}(\Omega) \cap C^{0, \gamma}(\Omega)$ and that the estimate $\left\|\tau_{\varepsilon}\right\|_{L^{\infty}(\Omega)} \leqslant M$ holds, independently of $\varepsilon$. Next, with this $\tau_{\varepsilon}$ and the same $\mathbf{w}$, define $\mathbf{w}_{\varepsilon}=\mathbf{u}\left(\tau_{\varepsilon}, \mathbf{w}\right)$ as the unique solution of the variational inequality (33) given by Proposition 1 and corresponding to the choice $a(t)=\frac{1}{\varepsilon} \chi_{\varepsilon}(t)$. Observe that $q>\frac{3 n}{n+2}$ implies $q>\frac{2 n}{n+2}$ and concerning $r, r>\frac{3 n}{n-1}>2$ and $r>\frac{3 n}{n-1}>\frac{n q}{n q+q-2 n}$, which is also implied by $q>\frac{3 n}{n+2}$. We have $\mathbf{w}_{\varepsilon} \in \mathrm{V}^{q}(\Omega)$, which is compactly imbedded in $L_{\sigma}^{s}(\Omega)$, if $s<\frac{q n}{n-q}$ and consequently also in $L_{\sigma}^{r}(\Omega)$. In fact

$$
\frac{3 n}{n-1}<\frac{q n}{n-q} \Leftrightarrow q>\frac{3 n}{n+2} \text { and } \frac{n}{p-1}<\frac{q n}{n-q} \Leftrightarrow q>\frac{n}{p}
$$

which are exactly the assumptions we made. We define $\mathscr{T}$ putting $\mathscr{T}(w)=\mathbf{w}_{\varepsilon}$ :

$$
\begin{aligned}
\mathscr{T}: L_{\sigma}^{r}(\Omega) & \rightarrow Z_{p} \times L_{\sigma}^{r}(\Omega) \rightarrow \mathbf{V}^{q}(\Omega) \subset L_{\sigma}^{r}(\Omega) \\
\mathbf{w} & \mapsto\left(\tau_{\varepsilon}(\mathbf{w}), \mathbf{w}\right) \mapsto \mathbf{w}_{\varepsilon} \mapsto \mathbf{w}_{\varepsilon}
\end{aligned}
$$

We now take an appropriate ball in $L_{\sigma}^{r}(\Omega)$ for domain of $\mathscr{T}$ :

$$
B_{p}=\left\{\mathbf{w} \in L_{\sigma}^{r}(\Omega):\|\mathbf{w}\|_{\left[L^{r}(\Omega)\right]^{n}} \leqslant \rho\right\},
$$

where $\rho$ is a constant, independent of $\mathbf{w}$ and $\varepsilon$, that comes from the following estimate:

$$
\left\|\mathbf{w}_{\varepsilon}\right\|_{\left[L^{r}(\Omega)\right]^{n}} \leqslant C_{r, q}\left\|\mathbf{w}_{\varepsilon}\right\|_{\mathbf{v}^{q}(\Omega)} \leqslant C_{r, q} C_{*} \equiv \rho
$$

where we first use the Sobolev imbedding $V^{q}(\Omega) \subset L_{\sigma}^{r}(\Omega)$ and then the estimate (34) in Remark 7. In particular, we have $\mathbf{w}_{\varepsilon}=\mathscr{T}(\mathbf{w}) \in B_{\rho}, \forall \mathbf{w} \in L_{\sigma}^{r}(\Omega)$ and so

$$
\mathscr{T}\left(B_{\rho}\right) \subset \mathscr{T}\left(L_{\sigma}^{r}(\Omega)\right) \subset B_{\rho} .
$$

It only remains to show that $\mathscr{T}$ is continuous and compact. Take a sequence $\mathbf{w}_{\delta} \rightarrow \mathbf{w}_{0}$ in $L_{\sigma}^{r}(\Omega)$-weak; from Proposition 3 we get $\tau_{\delta}\left(\mathbf{w}_{\delta}\right) \rightarrow \tau_{0}\left(\mathbf{w}_{0}\right)$ in $W^{1, p}(\Omega)$-weak and $L^{1}(\Omega)$-strong. Now the continuous dependence result in Proposition 1 implies that $\mathscr{T}\left(\mathbf{w}_{\delta}\right) \rightarrow \mathscr{T}\left(\mathbf{w}_{0}\right)$ strongly in $L_{\sigma}^{r}(\Omega)$.

We then apply the theorem of Schauder and obtain a solution of the approximated problem with the fixed point

$$
\mathbf{u}_{\varepsilon}=\mathscr{T}\left(\mathbf{u}_{\varepsilon}\right) \text { and } \theta_{\varepsilon}=\tau_{\varepsilon}\left(\mathbf{u}_{\varepsilon}\right) .
$$

The a priori estimates are consequences, respectively, of (38), (36), (37), and (34).

\section{EXISTENCE OF WEAK SOLUTIONS}

\section{Proof of the Theorem}

Using the compactness properties of the functional spaces involved, from the estimates obtained for the solution of the approximated problem, we extract subsequences such that, for $\varepsilon \rightarrow 0$, and $s \leqslant \frac{n p-p}{n-p}$ (recall $p<n$ ),

$$
\theta_{\varepsilon} \rightarrow \theta \text { in } W^{1, p}(\Omega) \text {-weak, } L^{p}(\Omega) \text {-strong, uniformly in any compact subset }
$$

$K \subset \Omega$ and the corresponding traces in $L^{s}\left(\Gamma_{\mathrm{N}}\right)$-strong;

$$
1-\chi_{\varepsilon}\left(\theta_{\varepsilon}\right)-\chi \text { in } L^{\infty}(\Omega) \text {-weak *; }
$$

$$
\mathbf{u}_{\varepsilon} \rightarrow \mathbf{u} \text { in } \mathbf{V}^{q}(\Omega) \text {-weak and in }\left[L^{s}(\Omega)\right]^{n} \text {-strong, for } s<\frac{q n}{n-q} \text {; }
$$

for some limit functions $\theta \in W^{1, p}(\Omega) \cap C^{0, \gamma}(\Omega)$ and $\chi \in L^{\infty}(\Omega)$ and $\mathbf{u} \in \mathbf{V}^{q}(\Omega)$. We use, as usual, the same index for the subsequences.

The fact that $\theta$ is continuous assures that the liquid and solid zones, defined respectively by

$$
\Lambda(\theta)=\{x \in \Omega: \theta(x)>0\} \text { and } \Sigma(\theta)=\{x \in \Omega: \theta(x)<0\},
$$

are open subsets of $\Omega$.

To prove (26), we observe that since $0 \leqslant \chi_{\varepsilon}\left(\theta_{\varepsilon}\right) \leqslant 1$, in the limit we also obtain $0 \leqslant \chi \leqslant 1$ a.e. in $\Omega$. From (41) and the fact that $\left(\theta_{\varepsilon}+\varepsilon\right)^{-} \rightarrow \theta^{-}$in $L^{1}(\Omega)$, due to (40), we obtain

$$
\int_{\Omega}\left[1-\chi_{\varepsilon}\left(\theta_{\varepsilon}\right)\right]\left(\theta_{\varepsilon}+\varepsilon\right)^{-} \rightarrow \int_{\Omega} \chi \theta^{-}
$$


But $\left[1-\chi_{\varepsilon}\left(\theta_{\varepsilon}\right)\right]\left(\theta_{\varepsilon}+\varepsilon\right)^{-}=0$ and then $\chi \theta^{-}=0$ a.e. in $\Omega$. We conclude that if

$$
\theta^{-}>0 \text { in } \Sigma(\theta) \text { then } \chi=0 \text { a.e. in } \Sigma(\theta) \text {, }
$$

and so $\chi \leqslant 1-\chi_{\Sigma(\theta)}$ a.e. in $\Omega$. To obtain $\chi \geqslant \chi_{\Lambda(\theta)}$, we repeat the above reasoning with $\theta_{\varepsilon}^{+}$and $\chi_{\varepsilon}\left(\theta_{\varepsilon}\right)$.

To prove (27), take any compact subset $K \subset \Sigma(\theta)$. We have $\max _{\mathbf{K}} \theta<0$ and the uniform convergence in (40) gives $1-\chi_{\varepsilon}\left(\theta_{\varepsilon}\right)=0$ in $K$ for $\varepsilon$ sufficiently small, say $\varepsilon<\varepsilon_{0}(K)$. Letting $\Psi=0$ in (32), we obtain

$$
\frac{1}{\varepsilon} \int_{K}\left|\mathbf{u}_{\varepsilon}\right|^{2} \leqslant \frac{1}{\varepsilon} \int_{\Omega} \chi_{\varepsilon}\left(\theta_{\varepsilon}\right)\left|\mathbf{u}_{\varepsilon}\right|^{2} \leqslant \max _{|\tau| \leqslant M}|f(\tau)| \int_{\Omega}\left|\mathbf{u}_{\varepsilon}\right| \leqslant C,
$$

for a constant $C$ which is independent of $\varepsilon<\varepsilon_{0}(K)$, by the a priori estimate on $\mathbf{u}_{\varepsilon}$. We then obtain $\mathbf{u}=0$ a.e. in $K$ and since $K$ is arbitrary also in $\Sigma(\theta)$. Observe that, in particular, $\mathbf{u}_{\varepsilon} \rightarrow \mathbf{u}$ in $\left[L^{2}(\Omega)\right]^{n}$-strong since $2<\frac{q n}{n-q}$ due to our assumptions on $q$.

We can now pass to the limit in the equation for the temperature, using the results of [11], having only in mind that, due to the a priori estimate $\left\|\theta_{\varepsilon}\right\|_{L^{\infty}(\Omega)} \leqslant M$ and the assumed convergence $b_{\varepsilon} \rightarrow b$, uniformly on compact sets, $b_{\varepsilon}\left(\theta_{\varepsilon}\right)$ is uniformly bounded and converges pointwise a.e. to $b(\theta)$ in $\Omega$. From (42) we get

$$
b_{\varepsilon}\left(\theta_{\varepsilon}\right) \mathbf{u}_{\varepsilon} \rightarrow b(\theta) \mathbf{u} \text { in }\left[L^{p^{\prime}}(\Omega)\right]^{n} .
$$

Recall that $q>\frac{n}{p}$ implies $\frac{q n}{n-q}>\frac{n}{p-1} \geqslant p^{\prime}$.

To finish the proof we just need to pass to the limit in the variational inequality and for this we just need to follow the procedure in [10]; we omit the details.

Acknowledgements-Partially supported by FCT(JNICT), PRAXIS XXI and PRAXIS/2/2.1/MAT/125/94 projects.

\section{REFERENCES}

1. J. Szekely, On some free and moving boundary problems in materials processing. In Free Boundary Problems: Theory and Applications (Edited by K. H. Hoffmann and J. Sprekels), pp. 222-242. Pitman Research Notes in Mathematics Series, 185 (1990)

2. B. Basu and J. Srinivasan, Numerical study of steady-state laser melting problem. Int. J. Heat Mass Transfer 31, 2331-2338 (1988).

3. C. J. Chang and R. A. Brown, Natural convection in steady solidification: finite element analysis of a two-phase Rayleigh-Bénard problem. J. Comput. Phys. 53, 1-27 (1994).

4. A. M. Meirmanov, The Stefan Problem. Walter de Gruyter, Berlin (1992).

5. R. Temam, Navier-Stokes Equations, 3rd revised edition. Elsevier, Amsterdam (1984).

6. G. Duvaut and J. L. Lions, Inequalities in Mechanics and Physics. Springer-Verlag, Berlin (1976).

7. D. Cioranescu, Quelques exemples de fluides Newtoniens généralisés. In Mathematical Topics in Fluid Mechanics (Edited by J. F. Rodriques and A. Sequeira), pp. 1-31. Pitman Research Notes in Mathematics Series, 274 (1992).

8. J. R. Cannon, E. DiBenedetto and G. H. Knightly, The steady-state Stefan problem with convection. Arch. Rational Mech. Anal. 73, 79-97 (1980).

9. J. F. Rodrigues, A steady-state Boussinesq-Stefan problem with continuous extraction. Annalli Mat. Pura et Appl. (iv) 144, 203-218 (1986).

10. J. F. Rodrigues, Weak solutions for thermoconvective flows of Boussinesq-Stefan type. In Mathematical Topics in Fluid Mechanics (Edited by J. F. Rodrigues and A. Sequeira), pp. 93-116. Pitman Research Notes in Mathematics Series, 274 (1992).

11. J. M. Urbano, A stationary Stefan problem with convection and non-linear diffusion. Portugaliae Mathematica 52(4), 481-497 (1995).

12. J. Mălek, K. R. Rajagopal and M. Ružička, Existence and regularity of solutions and stability of the rest state for fluids with shear dependent viscosity. Mathematical Models and Methods in Applied Science 5(6), 789-812 (1995).

13. F. Baldoni and K. R. Rajagopal, A continuum theory for the thermomechanics of solidification. Int. $J$. Non-Linear Mechanics. 32, 3-20 (1997).

14. O. Ladyzhenskaya and N. Ural'tseva, Linear and Quasi-linear Elliptic Equations. Academic Press, New York (1968).

15. J. L. Lions, Quelques Méthodes de Résolution des Problèmes aux Limites non Linéaires. Dunod, GauthierVillars, Paris (1969). 